\title{
Cinacalcet corrects biased allosteric modulation of CaSR by AHH autoantibody
}

\author{
Noriko Makita, ${ }^{1}$ Takao Ando, ${ }^{2}$ Junichiro Sato, ${ }^{1}$ Katsunori Manaka, ${ }^{1}$ Koji Mitani, ${ }^{1}$ Yasuko Kikuchi, ${ }^{3}$ \\ Takayoshi Niwa, ${ }^{3}$ Masanori Ootaki, ${ }^{4}$ Yuko Takeba, ${ }^{4}$ Naoki Matsumoto, ${ }^{4}$ Atsushi Kawakami, ${ }^{2}$ \\ Toshihisa Ogawa, ${ }^{5}$ Masaomi Nangaku, ${ }^{1}$ and Taroh liri ${ }^{1,4}$ \\ 'Department of Endocrinology and Nephrology, The University of Tokyo School of Medicine, Tokyo, Japan. 'Division of \\ Endocrinology and Metabolism, Nagasaki Graduate School of Biomedical Sciences, Nagasaki, Japan. ${ }^{3}$ Department of \\ Breast and Endocrine Surgery, The University of Tokyo School of Medicine, Tokyo, Japan. ${ }^{4}$ Department of Pharmacology, \\ St. Marianna University School of Medicine, Kawasaki, Japan. ${ }^{5}$ Breast Center, Dokkyo Medical University Koshigaya \\ Hospital, Saitama, Japan.
}

Biased agonism is a paradigm that may explain the selective activation of a signaling pathway via a GPCR that activates multiple signals. The autoantibody-induced inactivation of the calciumsensing receptor (CaSR) causes acquired hypocalciuric hypercalcemia (AHH). Here, we describe an instructive case of AHH in which severe hypercalcemia was accompanied by an increased CaSR antibody titer. These autoantibodies operated as biased allosteric modulators of CaSR by targeting its Venus flytrap domain near the $\mathrm{Ca}^{2+}$-binding site. A positive allosteric modulator of CaSR, cinacalcet, which targets its transmembrane domain, overcame this autoantibody effect and successfully corrected the hypercalcemia in this patient. Hence, this is the first study to our knowledge that identifies the interaction site of a disease-causing CPCR autoantibody working as its biased allosteric modulator and demonstrates that cinacalcet can correct the AHH autoantibody effects both in vitro and in our AHH patient. Our observations provide potentially new insights into how biased agonism works and how to design a biased allosteric modulator of a CPCR. Our observations also indicate that the diagnosis of AHH is important because the severity of hypercalcemia may become fatal if the autoantibody titer increases. Calcimimetics may serve as good treatment options for some patients with severe AHH.

Authorship note: N. Makita and TA contributed equally to this work

Conflict of interest: MN receives lecture fees and research grants from Kyowa Hakko Kirin.

Copyright: () 2019 American Society for Clinical Investigation

Submitted: November 27, 2018 Accepted: March 12, 2019 Published: April 18, 2019

Reference information: /CI Insight. 2019;4(8):e126449. https://doi. org/10.1172/jci.insight.126449.

\section{Introduction}

Biased agonism (1-8), especially when involving an allosteric modulation (9-13), has generated much interest in the field of GPCR regulation, both in terms of its physiology and pharmacology, because it may enable the selective activation of a signaling pathway (i.e., a desirable signal only) via a GPCR with diverse signaling properties.

Achieving the selective activation of a particular GPCR signal is likely to be important for a cell to function properly (14-16). Classically, each GPCR was believed to activate a specific G protein and was classified accordingly (Gs-, Gi-, Gq-, or G13-coupled GPCRs: i.e., GPCRs that couple to Gs, Gi, Gq, or G13, respectively). However, when GPCR genes were subsequently cloned and studied, many examples of signal divergence were found in which a particular GPCR activated many G proteins and therefore multiple downstream signaling pathways (14-16). The principal question that then arose from this discovery was how selective activation of a particular signal could be enabled via a "selectivity-loose" GPCR that activates multiple signals. Biased agonism emerged as one possible mechanism (1-8). Some biased agonists (often pharmacological agonists) can stabilize a unique active conformation of a GPCR that activates one G protein-dependent signal only. This differs from the usual (nonbiased) agonist-dependent activation of a GPCR that would enable the activation of all of its target $G$ proteins. Biased agonism using an allosteric modulator is a striking example of this (9-13), because in this case even a usual (nonbiased) agonist enables biased agonism in the presence of a biased allosteric modulator. Especially, if this operates in a cell, either physiologically (17) or pathophysiologically, the presence of an appropriate biased allosteric modulator based on a preexisting nonbiased system (i.e., a combination of a nonbiased agonist and a GPCR that activates multiple $G$ proteins) would be alone sufficient for a desired biased agonism mechanism to function. 
This will be more effective than expressing a GPCR that selectively activates one signaling pathway because protein expression would be a more time- and energy-consuming process within the cell.

Calcium-sensing receptor (CaSR), a member of the GPCR family c, plays a pivotal role in maintaining extracellular calcium homeostasis (18-25). CaSR is activated by extracellular calcium and activates multiple $\mathrm{G}$ proteins, including $\mathrm{Gq} / 11$ and $\mathrm{Gi} / \mathrm{o}$, and negatively regulates the secretion of parathyroid hormone in parathyroid epithelial cells and the reabsorption of calcium in renal tubular epithelial cells (18-25).

Heterozygous inactivating mutations of CaSR cause familial hypocalciuric hypercalcemia (FHH) (26), which typically involves slight hypercalcemia and hypocalciuria and requires no particular treatment. For this reason, FHH used to be known as familial benign hypocalciuric hypercalcemia. In contrast, homozygous inactivating mutations of CaSR cause neonatal severe hyperparathyroidism (NSHPT), which leads to fatal hypercalcemia if a subtotal parathyroidectomy is not performed (26).

Inactivating autoantibodies directed against CaSR cause acquired hypocalciuric hypercalcemia (AHH) (27-29), which was first described by Pallais et al. (30). In a case of AHH, we subsequently found unique autoantibodies that operate as biased allosteric modulators of CaSR, thereby augmenting Gq/IP signaling pathway and yet attenuating the Gi/ERK1/2 signaling pathway (29, 31). Recently, Pallais et al. have also described a patient whose autoantibodies attenuated ERK1/2 phosphorylation but showed no effect against inositol phosphate (IP) accumulation (32). Taken together, these findings suggest that, not only Gq/11 (19, 33-35), but also Gi/o (19, 29, 31), is likely to play an important role in CaSR signaling.

FHH and NSHPT are largely discrete and independent diseases. In contrast, inactivating autoantibodies directed against CaSR can vary in the severity of their effects from a mild FHH level to a severe NSHPT level. In addition, logically, as the titer and character of these autoantibodies changes, the severity of hypercalcemia may change from mild levels to potentially fatal levels during the clinical course of a single patient. The diagnosis and treatment of severe hypercalcemia is clinically very important and yet we speculate that AHH may often have been missed, misdiagnosed, or underestimated. Here, we describe an instructive case of severe $\mathrm{AHH}$ in which the serum calcium level fluctuated from permissive ranges to high levels as the CaSR autoantibody titer increased. Through an analysis of the CaSR autoantibody in this patient, we have, for the first time to our knowledge, identified the interaction site of a disease-causing GPCR autoantibody that works as its biased allosteric modulator, which may provide insight into the molecular mechanism of biased allosteric modulation $(12,36,37)$. In addition, based on our in vitro analyses, we have successfully treated this $\mathrm{AHH}$ patient with a positive allosteric modulator, cinacalcet $(36,38)$.

\section{Results}

Identification of an autoantibody against CaSR in the patient's serum. We assessed a male patient with hypercalcemia and hypocalciuria, who was clinically diagnosed as having AHH (Figure 1A; see Methods for a full description; and Supplemental Figure 1; supplemental material available online with this article; https:// doi.org/10.1172/jci.insight.126449DS1). The patient's sera (Figure 1A, June 2010, arrow I) were found to react against HEK293 cells transiently expressing human CaSR (Figure 1B, left), indicating that the patient harbored autoantibodies in his serum against CaSR. This was confirmed by double immunofluorescence cytochemistry, showing colocalization of patient IgG with monoclonal anti-CaSR antibodies in HEK293 cells expressing CaSR stably (Figure 1B, right).

We then evaluated how the patient's autoantibodies would affect the CaSR signaling pathway. Interestingly, we observed that the patient's autoantibodies work as biased allosteric modulators of CaSR, in a manner that we had reported previously $(29,31)$. The patient IgG paradoxically potentiated the CaSR-mediated accumulation of IP, while inhibiting the CaSR-mediated phosphorylation of ERK1/2 (Figure 2A). With up to $2.5 \mathrm{mM} \mathrm{Ca}^{2+}$, phosphatidylinositol (PI) turnover or ERK1/2 phosphorylation was almost completely dependent on $\mathrm{Gq} / 11$ or Gi/o, respectively (data not shown), as previously reported (31).

A spontaneous exacerbation successfully corrected by cinacalcet. In December 2010, the patient's calcium level increased up to $15 \mathrm{mg} / \mathrm{dl}$. In parallel, it was found that his autoantibody titer had spontaneously increased (Figure 1A, right). We suspected that calcimimetics might be one of the treatment options. We found in vitro that cinacalcet, a positive allosteric modulator of CaSR, can overcome the biased allosteric modulation of CaSR by our patient's autoantibodies, by increasing PI turnover and ERK1/2 phosphorylation (Figure 2B). It is noteworthy that cinacalcet did not inhibit the binding of our patient's autoantibodies to CaSR (Figure 2B, inset). Prompted by these findings, we successfully treated our patient with cinacalcet (Figure 1A; please also see full patient description (see Methods for 
A
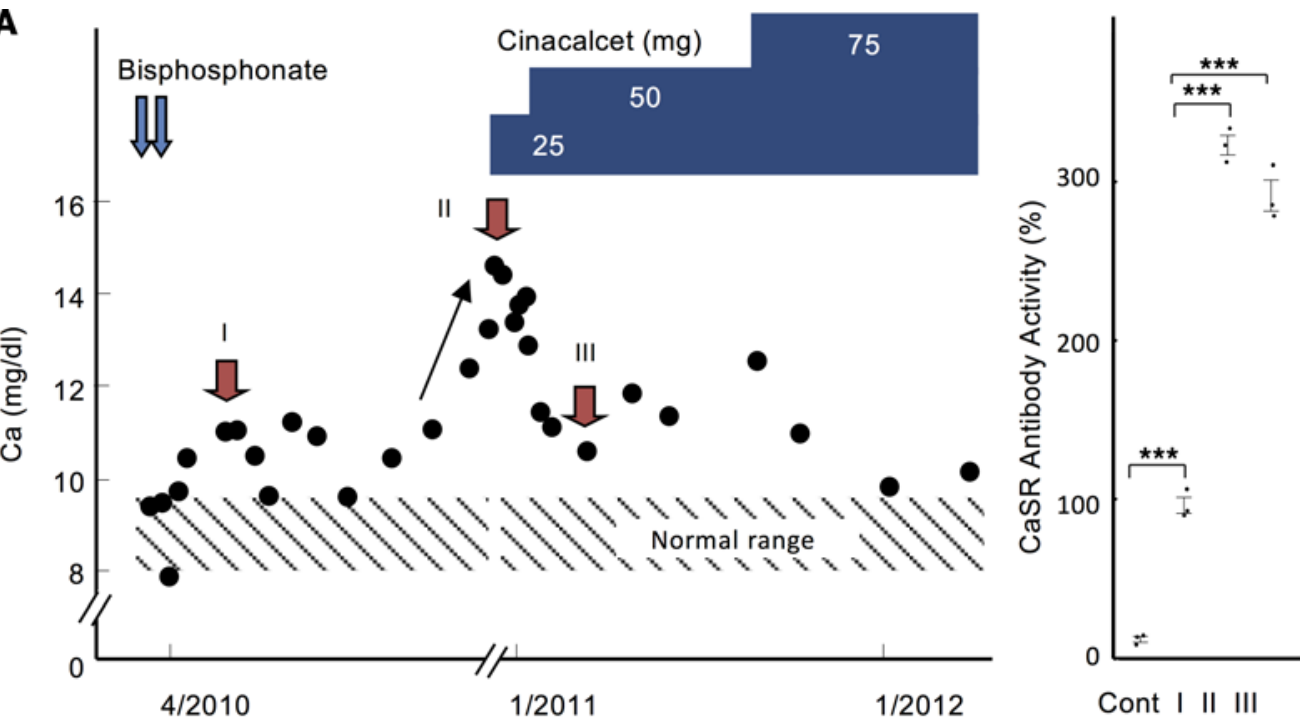

B
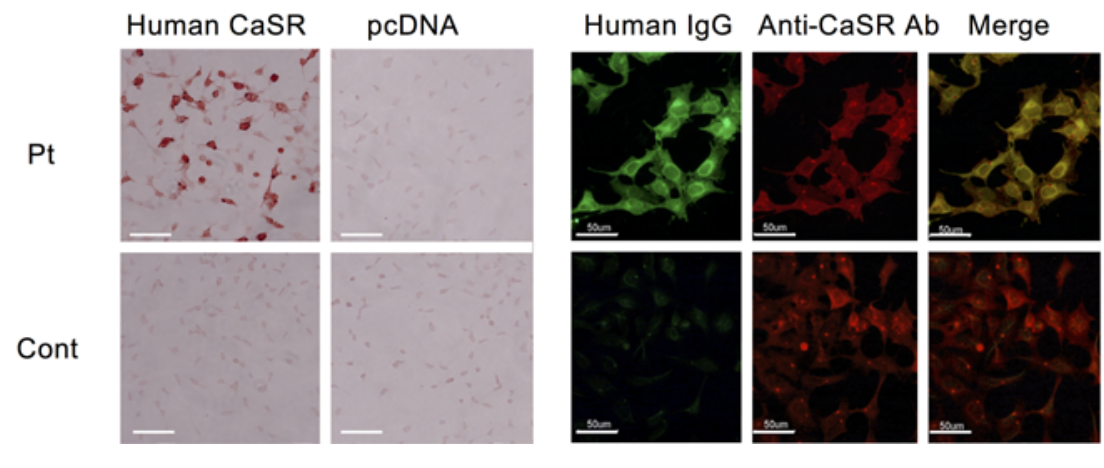

Figure 1. Temporal profile of serum Ca levels in our AHH patient and identification of CaSR autoantibody. (A) Left: The corrected calcium levels in our patient's serum over time. Shortly after the episode of spontaneous exacerbation of hypercalcemia, treatment with cinacalcet was started. Right: CaSR antibody activities before (arrows I and II) and after (arrow III) the treatment. Values represent mean \pm SEM of triplicate determinations. Each set of results is representative of at least 2 additional experiments. Statistical analysis was performed using 2-way ANOVA with Dunnett's test. ${ }^{* * *} P<$ 0.0001. (B) Left: Immunoperoxidase staining of HEK293 cells expressing human CaSR incubated with the AHH patient/ control sera (1:50). Right: Colocalization (merge: yellow) of the AHH patient autoantibody (green) with monoclonal antiCaSR (amino acids 15-29) antibody (red) was observed. Scale bar: $50 \mu \mathrm{m}$.

full patient description). Here, it is of note that his autoantibody titer did not spontaneously decrease during this treatment (Figure 1A, right). These data suggested that the improvement of hypercalcemia was not spontaneous but due to the effects of cinacalcet.

Augmentation of PTH secretion by the patient IgG and pertussis toxin. In human parathyroid cells derived from a resected parathyroid adenoma, the serum from our patient (1:20) indeed augmented PTH secretion (Figure 2C, middle). Furthermore, pertussis toxin (PTX) treatment augmented PTH secretion (Figure $2 \mathrm{C}$, right), lending further support to our finding that not only $\mathrm{Gq} / 11(19,33,34)$, but also Gi/o $(19,29,31)$, plays a role in regulating PTH secretion.

Molecular mode of action of the CaSR autoantibody. The main scientific question that arises from our present case study is where and how our patient's autoantibodies can effect the CaSR. We initially suspected that our patient's autoantibodies might interact with the transmembrane domains of CaSR based on the fact that both calcimimetics and calcilytics interact with these domains. However, we unexpectedly discovered that these autoantibodies interact with the flytrap domain of CaSR. Immunofluorescence assays without permeabilization (Figure 3A) showed that the autoantibodies recognized the extracellular domain (ECD) of CaSR. ELISA (39) did not identify any synthetic peptide derived from the ECD that interacted with the autoantibodies (data not shown), suggesting that the autoantibodies are conformational. We next assessed whether these autoantibodies might alter the 
A $\quad \begin{array}{ccc}\mathrm{EC}_{50} & \text { Cont } & 2.48 \pm 0.01 \mathrm{mM} \\ \mathrm{Pt} & 1.89 \pm 0.04 \mathrm{mM}^{\star *}\end{array}$

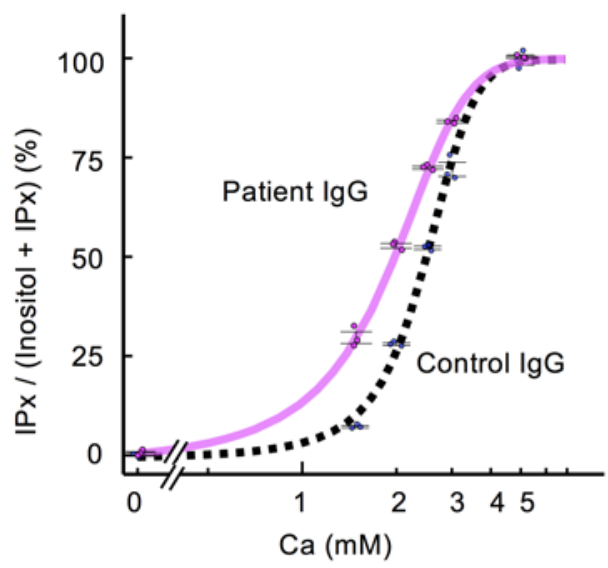

B

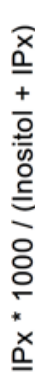

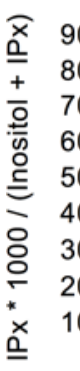

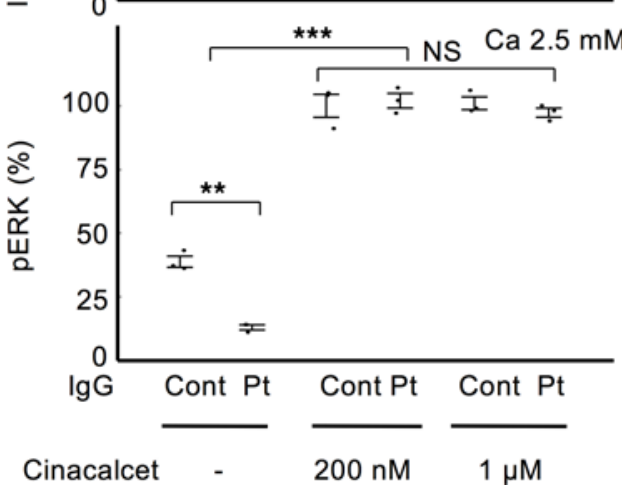

$\begin{array}{ccc}\mathrm{EC}_{50} & \text { Cont } & 2.61 \pm 0.03 \mathrm{mM} \\ \mathrm{Pt} & 3.75 \pm 0.20 \mathrm{mM}^{* *}\end{array}$

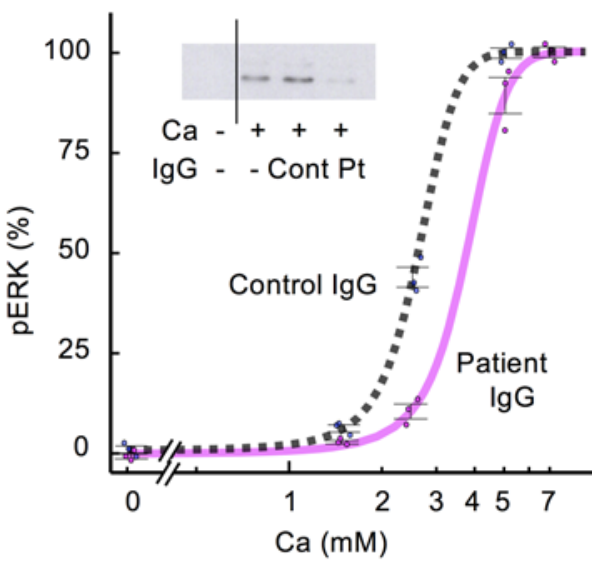

C

Ca $1.5 \mathrm{mM}$

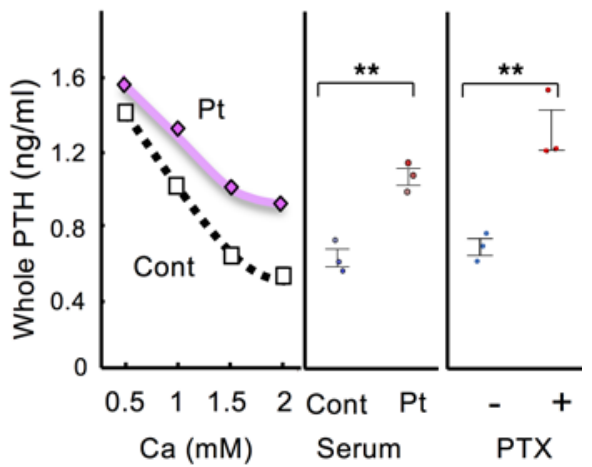

Figure 2. Identification of CaSR autoantibody working as a biased allosteric modulator to CaSR. (A) Effects of our AHH patient autoantibody (1:5) on CaSR-dependent PI turnover (left) and ERK1/2 phosphorylation (right) in HEK293 cells stably expressing CaSR stimulated by different concentrations of calcium ions. $\mathrm{EC}_{50}$ of calcium concentration \pm SEM in the presence of control IgC or the patient IgC was $2.48 \pm 0.01 \mathrm{vs} .1 .89 \pm 0.04 \mathrm{mM}$ (**P<0.01) (left) and $2.61 \pm$ 0.03 vs. $3.75 \pm 0.20 \mathrm{mM}$ ( ${ }^{* *} P<0.01$ ) (right). Statistical analysis was performed using Student's $t$ test. Values represent mean \pm SEM of triplicate determinations. Each set of results is representative of at least 2 additional experiments. (B) Effects of cinacalcet on these AHH patient autoantibody effects. Statistical analysis was performed using 2-way ANOVA with Tukey's multiple comparison test. Values represent mean \pm SEM of triplicate determinations. Each set of results is representative of at least 2 additional experiments. Scale bar: $50 \mu \mathrm{m}$ (inset). (C) Effects of the AHH patient sera (1:20 dilution) (left) and pertussis toxin (PTX) (right) on PTH secretion from parathyroid adenoma cells. Statistical analysis was performed using Student's $t$ test. Values represent mean \pm SEM of triplicate determinations. Each set of results is representative of at least 2 additional experiments. ${ }^{* *} P<0.01 ;{ }^{* *} P<0.0001$

dimerization or localization of CaSR. However, incubation with the patient's autoantibodies did not affect the dimerization state of CaSR (Figure 3A, right). It has been reported that the family c GPCRs are constitutive dimers, which can be converted to monomers under reducing condition $(25,40,41)$. Consistently, almost all of the CaSR was detected at sizes of between $205 \mathrm{kDa}$ and $300 \mathrm{kDa}$ (expected for dimeric CaSR) without DTT but around $150 \mathrm{kDa}$ with DTT (expected for monomeric CaSR). 
A

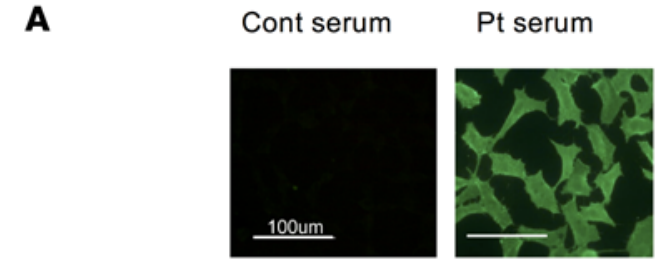

Pt serum
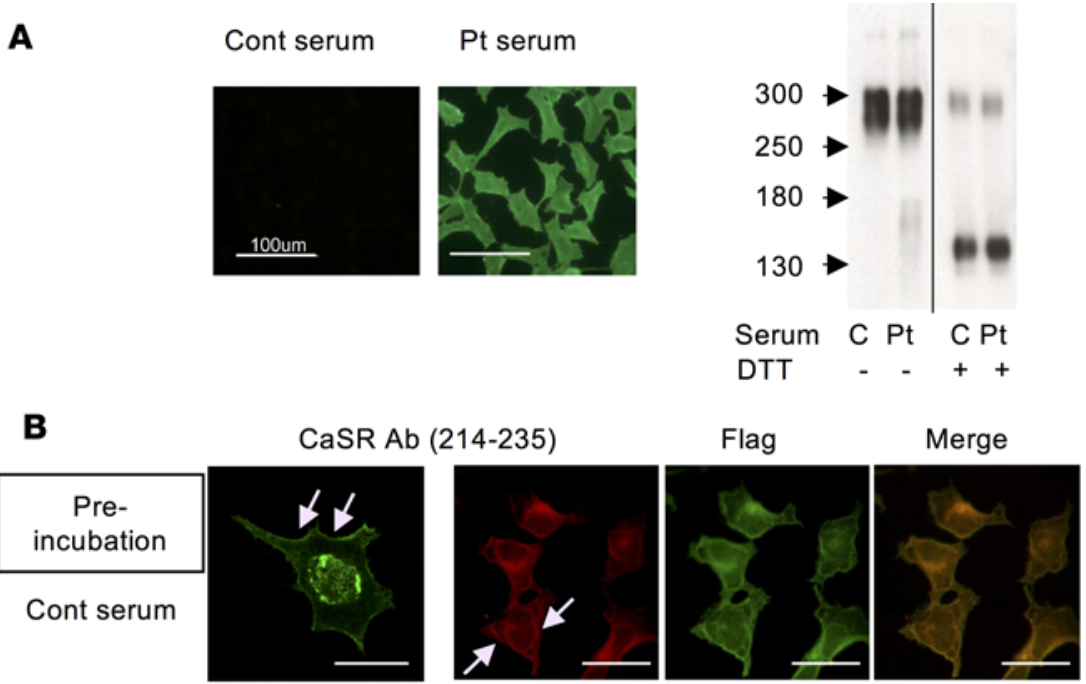

Pt serum
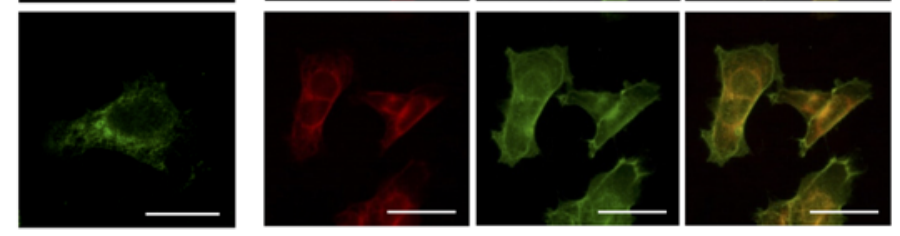

CaSR

Flag-CaSR
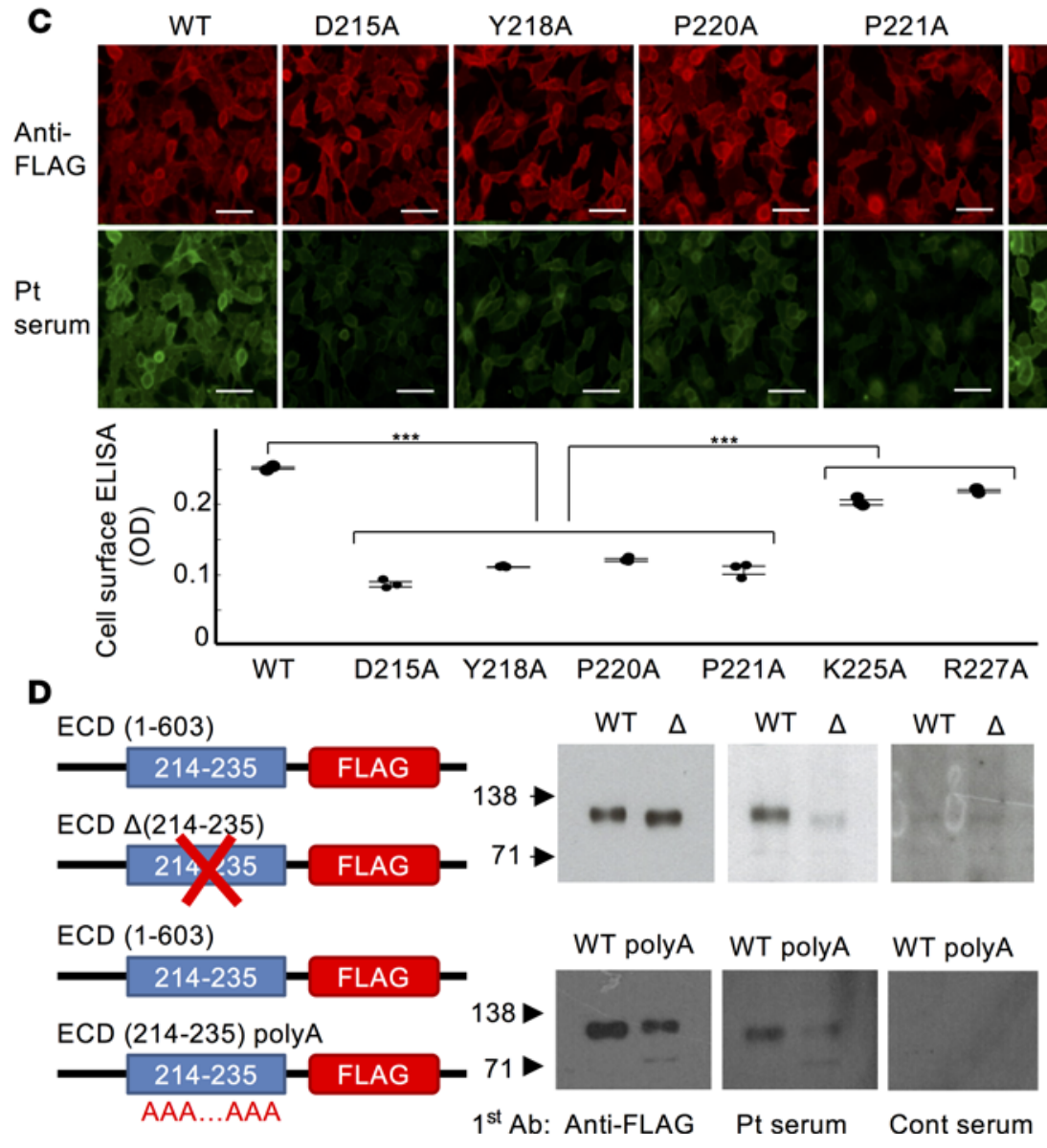
Figure 3. The patient autoantibody interacting with Venus flytrap domain of CaSR. (A) Left: Immunofluorescence images using the AHH patient sera without permeabilization. Scale bar: $100 \mu \mathrm{m}$. Right: Immunodetection of CaSR. Flag-tagged 293 CaSR cells were preincubated with patient's serum or control serum (1:50) at $37^{\circ} \mathrm{C}$ for 4 hours in medium containing $1.5 \mathrm{mM}$ calcium. They were then treated with $0.5 \mathrm{mg} / \mathrm{ml}$ EZ-link Sulfo-NHS-Biotin prior lysing in the presence of $100 \mathrm{mM}$ iodoacetamide. The CaSR was immunoprecipitated with anti-Flag antibody, eluted with SDS-sample buffer with or without DTT, and subjected to SDS-PAGE (5\%). CaSR on the cell surface was determined using streptavidin. (B) Localization of monoclonal anti-CaSR (amino acids 214-235) antibody (left) and colocalization of monoclonal anti-CaSR antibody and anti-Flag antibody (right) were investigated after incubation with control or the AHH patient sera (1:50). In plasma membrane images arrows indicate anti-CaSR (amino acids 214-235) antibody, which disappeared after incubation with the AHH patient sera (bottom). Scale bar: $25 \mu \mathrm{m}$. (C) Immunofluorescence images with the AHH patient sera were investigated in HEK293 cells expressing WT or mutant Flag-tagged CaSR. Values represent mean \pm SEM of triplicate determinations. Each set of results is representative of at least 2 additional experiments. Statistical analysis was performed using ANOVA with Dunnett's test. ${ }^{*} P<0.05 ;{ }^{* *} P<0.01 ;{ }^{* *} P<0.0001$. Scale bar: 50 $\mu \mathrm{m}$. (D) Construction of extracellular domain of CaSR (left) and its immunodetection using our AHH patient sera.

In contrast, and interestingly, incubation with the patient autoantibodies greatly altered the cognition of CaSR by a commercial CaSR antibody (Figure 3B, left). After incubation with our patient's serum (1:50) for 1 hour at $37^{\circ} \mathrm{C}$, immunofluorescence signals by a monoclonal anti-CaSR antibody (indicated by Figure 3B, arrows), which recognizes amino acids 214-235 in the protein (anti-214-235 monoclonal antibody), disappeared from the plasma membrane of HEK293-CaSR cells (Figure 3B, bottom left). Control serum did not have this effect when added to the culture medium (1:50) (Figure 3B, top left). These data prompted us to speculate that these autoantibodies might alter the localization of CaSR, possibly by stimulating internalization of CaSR. However, in HEK293 cells stably expressing Flag-tagged CaSR, after incubation with the patient's serum (1:50), immunofluorescence signals for the anti-CaSR (amino acids 214-235) monoclonal antibody (Figure 3B, top second image, arrows) disappeared from the plasma membrane (Figure 3B, bottom right), whereas those for the anti-Flag antibody were detectable (Figure 3B, right). These data instead suggested that the CaSR epitope recognized by our patient's autoantibodies may overlap with amino acids 214-235. It is here of note that the anti-CaSR (amino acids 214-235) monoclonal antibody affected neither CaSR-mediated IP accumulation nor CaSR-mediated ERK1/2 phosphorylation (Supplemental Figure 2). Furthermore, immunofluorescence analysis showed that the patient's serum reacted less with several mutants of CaSR with an alanine substitution at this region (D215A, Y218A, R220A, and P221A) than WT CaSR, whereas anti-Flag antibody reacted similarly with all mutants and WT CaSR (Figure 3C) (ELISA was the most sensitive method, although it showed higher background signals). Moreover, immunodetection of the ECD of Flag-tagged CaSR by the patient's serum mostly disappeared when amino acids 214-235 were deleted (Figure 3D, top) or mutated to alanines (Figure $3 \mathrm{D}$, bottom). Taken together, these data support the notion that our patient's autoantibodies recognize the region around amino acids 214-235, within the ECD of CaSR $(24,42,43)$ (Figure 4A).

\section{Discussion}

Here, we describe an instructive case of $\mathrm{AHH}$, who shows severe hypercalcemia accompanied by an increased CaSR antibody titer. The patient's autoantibodies operate as biased allosteric modulator of CaSR, notably by targeting its Venus flytrap domain (VFT) near the $\mathrm{Ca}^{2+}$-binding site (Figure 3 and Figure 4A) (12, 19, 24, 27, 43). To our knowledge, this is the first report that identifies the GPCR epitope that is recognized by GPCR autoantibodies operating as biased allosteric modulators. In addition, based on our in vitro analyses, we have succeeded in overcoming the effects of biased allosteric modulators, in this case autoantibodies against CaSR, through treatment with a positive allosteric modulator, cinacalcet (Figure 2B and Figure 4B). Study of the autoantibody-CaSR complex may help improve understanding of how biased agonists work and understanding of how to design a biased allosteric modulator of GPCRs that may switch on a desirable signal only.

In the case of $\mathrm{AHH}$, unlike $\mathrm{FHH}$, the degree of hypercalcemia may logically vary widely among patients from mild to severe levels, depending on the potency of the autoantibodies in suppressing CaSR signaling. In addition, the levels of hypercalcemia can fluctuate, even during the clinical course of an $\mathrm{AHH}$ patient, such as our current case (Figure 5). Therefore, we should try to identify AHH patients and be prepared for their treatment. As reported previously, glucocorticoids are a treatment option for AHH but do not always work $(30,32)$. Based on an in vitro analysis of the CaSR autoantibody, here we have successfully treated a patient with severe $\mathrm{AHH}$ with cinacalcet (Figure 1A).

To our knowledge, this is also the first report that demonstrates that cinacalcet can correct the $\mathrm{AHH}$ autoantibody effects both in vitro and in an AHH patient. In addition to Brown and colleagues (30), we speculated (31) that AHH patients might be successfully treated with calcimimetics, although Brown's group (30) noted that it was unclear whether calcimimetics could overcome the AHH autoantibody effects. We subse- 
A

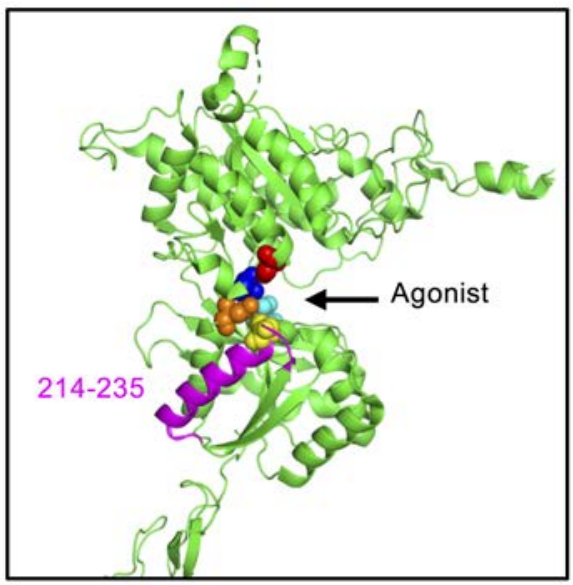

B

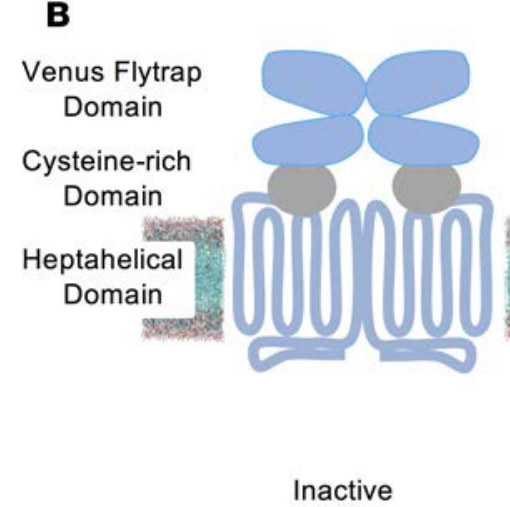

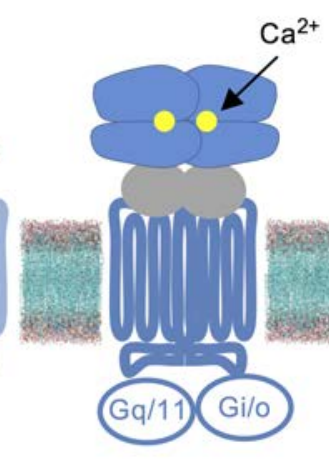

Active

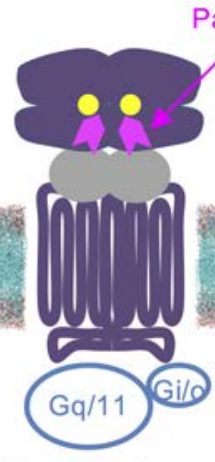

Biased active
Patient IgG

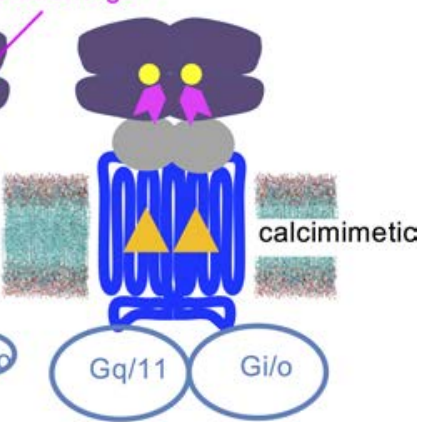

Active

(Corrected)

Figure 4. Model of activation and allosteric modulation of CaSR. (A) Illustration of the structure of the extracellular Venus flytrap domain (VFT) of the human CaSR (43). Amino acids 214-235 (magenta ribbon), Ca+-binding site, and key amino acids for Ca ${ }^{2+}$ binding are highlighted (S147, red; S170, blue; D190, yellow; Y218, cyan; and E297, orange) using PyMOL. (B) Illustration of the activation/allosteric modulation model of CaSR. At the resting inactive state, CaSRs form constitutive dimers. $\mathrm{Ca}^{2+}$ binding stabilizes the active dimer conformation, facilitating $\mathrm{Gq} / 11$ and $\mathrm{Gi} / \mathrm{o}$ activation. Ca ${ }^{2+}$ plus the $\mathrm{AHH}$ patient autoantibodies, which interact with VFT near the Ca ${ }^{2+}$-binding site, stabilize the biased active conformation of CaSR, enabling Gq/11-specific activation. A calcimimetic, cinacalcet, which targets its heptahelical transmembrane domain (closer to the $\mathrm{G}$ protein-coupling domain), overcomes the autoantibody effect, enhancing $\mathrm{Gq} / 11$ and $\mathrm{Gi} / \mathrm{o}$ activation and thereby successfully correcting the hypercalcemia in our AHH patient (see also Figure1A).

quently reported that a calcimimetic, NPS-R-568, was able to overcome the autoantibody effect of an AHH patient in intact cells (31). Kuo et al. recently reported in a patient with $\mathrm{AHH}$ and CKD that the increased serum PTH and Ca levels were corrected by cinacalcet treatment (44), although they did not confirm the effect of cinacalcet on the AHH autoantibody in in vitro assays. It is here of note that cinacalcet may currently serve as a nonspecific treatment option for hypercalcemia (45-49). Accordingly, an in vitro analysis is essential to conclude that cinacalcet can overcome the AHH autoantibody effect.

We have found in our present case study that the patient's autoantibodies recognize an epitope near the $\mathrm{Ca}^{2+}$-binding site of CaSR (Figure 3, B-D, and Figure 4) (12, 19, 24, 27, 43). In contrast, classical calcimimetics and calcilytics have been shown to operate at the 7-transmembrane (7TM) domain of CaSR (50), which is closer to the $\mathrm{G}$ protein-coupling domain. Importantly, cinacalcet did not inhibit the binding of our patient's autoantibodies to CaSR (Figure 2A, right, inset). Collectively, these findings may well explain why the positive allosteric modulator, cinacalcet, can overcome a biased allosteric modulator i.e., our patient's antibody (Figure 4B).

The question of how our patient's autoantibodies react with VFT and manipulate CaSR signaling, especially in a biased fashion, is a central issue in our current analyses, one for which we do not have the answer at the moment. In the case of umami or sweet taste receptors, composed of T1R1/T1R3 or T1R2/T1R3, several kinds of allosteric modulators that react with VFT or cysteine-rich domain besides 7-transmembrane domain have now been reported (51-53). In particular, allosteric modulators reacting with VFT act as a molecular glue near to the opening of the VFTs, stabilizing their closed and active conformation by strengthening the hydrophobic and electrostatic interactions between the upper and lower lobes and lowering the entropic pen- 


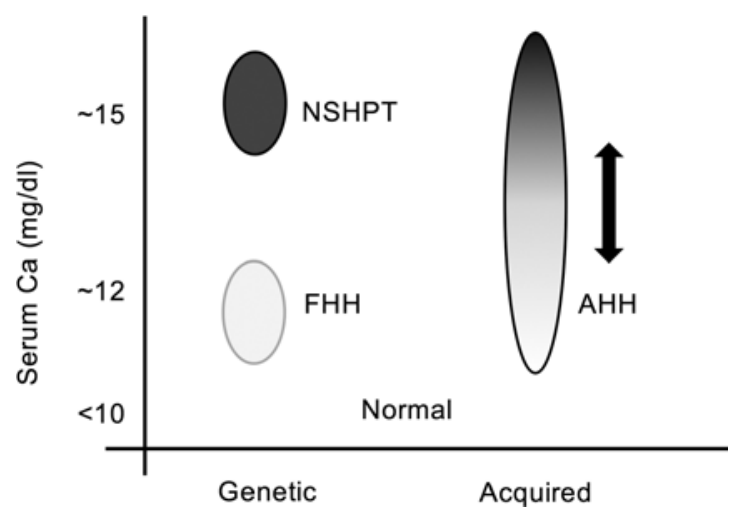

Figure 5. Genetic and acquired diseases caused by molecular defects of CaSR. Heterozygous inactivating mutations of CaSR cause familial hypocalciuric hypercalcemia $(\mathrm{FHH})$, which typically leads to slight hypercalcemia and hypocalciuria and requires no treatment. Homozygous inactivating mutations of CaSR cause neonatal severe hyperparathyroidism (NSHPT), which leads to fatal hypercalcemia without a subtotal parathyroidectomy. Thus, FHH and NSHPT are largely discrete and independent diseases. Inactivating autoantibodies directed against CaSR cause acquired hypocalciuric hypercalcemia (AHH). In the case of $\mathrm{AHH}$, the degree of hypercalcemia may vary widely among patients from mild FHH levels to severe NSHPT levels, depending on the potency of the autoantibodies. In addition, the levels of hypercalcemia can fluctuate, even during the clinical course of a single AHH patient, as the titer and character of these autoantibodies change.

alties of lobe closure. These factors cannot bind or stabilize the closed conformation of VFT in the absence of the agonist (as in our present case). In the case of CaSR, L-amino acids are known to be endogenous allosteric modulators $(19,20)$, which react with the agonist-binding pocket of VFT. Several key sites in the calcium-binding pocket of VFT have been identified by mutagenesis, chimera studies, and molecular modeling based on homology to mGluR1, for which the crystal structure has been already resolved $(40,41,54-58)$. In addition, recently, the crystal structure of the ECD of CaSR has been resolved (43), although it may be still controversial. Five CaSR sites in particular, S147, S170, D190, Y218, and E297, are conserved among family c GPCRs (59). Moreover, Y218, where a naturally occurring inactivating CaSR mutation was identified, is contained within the epitope that reacts with our patient's autoantibodies (Figure 3C and Figure 4A). Aromatic L-amino acids have been reported to enhance calcium-stimulated signals by binding to sites associated with calcium-binding sites (60). Interestingly, Pin's group has recently reported that a nanobody interacting with the VFT domain of the mGlu2 receptor acts as its positive allosteric modulator (61). It is thus possible that our patient's autoantibodies modulate CaSR signaling by reacting with a site near to one of the residues that is important for calcium binding. It is likely that our patient's autoantibodies affect the relative movement of the VFT domains, an essential step in the activation process of this class c GPCR (61).

An aspect of our current case that we believe to be unique is that the autoantibodies are not positive/ negative allosteric modulators (allosteric activators/inhibitors) but biased allosteric modulators, which augment one signal but suppress another. In fact, it has been reported that some allosteric modulators manipulate each aspect of CaSR signaling to different extents $(12,36,37)$. However, there have been no modulators reported that manipulate CaSR signaling in opposing directions $(12,36,37)$. If we can in the future identify the structural conformation of the epitope that the patient's autoantibodies react with and the mechanism of how they induce the unique activated form of CaSR in our current case, it may have a remarkable effect in increasing our knowledge of how biased agonists react with and modulate GPCRs, how GPCRs work physiologically or pathophysiologically, and how to design biased allosteric modulators of GPCRs $(9-11,62)$ that may switch a desirable signal "on" and an undesirable signal "off."

\section{Methods}

Patient information. An 83-year-old man who had been previously in good health visited a hospital in 2009 with appetite loss and was found to be suffering from hypercalcemia and hypophosphatemia with hypocalciuria (cCa $16.5 \mathrm{mg} / \mathrm{dl}$, normal 8.4-9.7, inorganic phosphate (iP) $1.4 \mathrm{mg} / \mathrm{dl}$, FECa 0.04\%). His intact PTH level was slightly elevated $(83 \mathrm{pg} / \mathrm{ml}$, normal $10-65 \mathrm{pg} / \mathrm{ml})$, and there was no detectable PTH-related protein. There was no renal insufficiency (Cr $0.7 \mathrm{mg} / \mathrm{dl})$. He was treated intravenously with bisphosphonate and elcitonin. His calcium 
levels were maintained at around $13 \mathrm{mg} / \mathrm{dl}$ under bisphosphonate treatment and then tended to decrease.

In April 2010, the patient showed muscle weakness due to transient hypocalcemia and was referred to our hospital (Figure 1A). The patient's calcium levels have been around $11 \mathrm{mg} / \mathrm{dl}$ without bisphosphonate treatment since then. Neither ultrasonography nor ${ }^{99 \mathrm{~m} T c-M I B I}$ scintigraphy identified adenoma/hyperplasia at the parathyroid sites (63-66). He continued to show hypophosphatemia (iP $1.5 \mathrm{mg} / \mathrm{dl}$ ) and hypocalciuria (FECa $<1 \%)$. Based on his laboratory data and clinical course of spontaneous improvement, we diagnosed that he suffered from $\mathrm{AHH}$ resulting from an autoantibody that inactivates CaSR. The patient had no familial history of hypercalcemia. The patient had no personal history of other autoimmune diseases either. In addition, the patient had no mutations in the CASR, GNA11, AP2S1, or GCM2 genes.

In December 2010, however, his calcium level increased again up to $15 \mathrm{mg} / \mathrm{dl}$. Concerning the treatment of severe $\mathrm{AHH}$, it has been reported that glucocorticoids are effective in some cases (30) but not effective in others (32). Moreover, we suspected that our patient was old enough to become easily immunocompromised by moderate or high doses of glucocorticoid. We further suspected that calcimimetics might be one of the treatment options. After confirming that cinacalcet overcomes the effect of our patient's autoantibody, we treated our patient with $25 \mathrm{mg}$ cinacalcet, which was subsequently increased to $75 \mathrm{mg}$. Following this therapy, our patient's calcium levels decreased to around $10 \mathrm{mg} / \mathrm{dl}$ without any side effects (Figure 1A). This therapeutic effect persisted for years thereafter (Supplemental Figure 1).

Sample preparation and DNA sequencing. Serum samples were collected and stored at $-80^{\circ} \mathrm{C}$ until just before use. Purified IgG was isolated using a Montage antibody purification kit (MilliporeSigma), followed by concentration using Amicon Ultra 15 (MilliporeSigma) for immediate assay (31). Genetic analysis of CASR, GNA11, AP2S1, and GCM2 genes and other experiments using patient samples, including serum or parathyroid, were performed under approval of the Institutional Review Board of the University of Tokyo.

Expression constructs, cell culture, and transfection. HEK293 cells (obtained from Henry R. Bourne, UCSF, San Francisco, CA, USA), maintained in DMEM containing 10\% FBS, were transfected with constructs encoding human CaSR or Flag-tagged WT/mutant human CaSR, using Lipofectamine 2000 (Invitrogen) $(31,67,68)$. The transiently transfected cells were used for immunoperoxidase staining and mutant study after 48 hours in culture. Otherwise, we used HEK293 cells stably expressing human CaSR or flag-tagged human CaSR, selected, and cloned in medium containing $0.8 \mathrm{mg} / \mathrm{ml} \mathrm{G} 418$ as described previously (31, 69-71). Plasmid expressing only the ECD of CaSR (amino acids 1-603) was made by subcloning PCR product using Flag-tagged CaSR in pcDNA3.1 as a template. Mutagenesis of CaSR or the ECD domain of CaSR (amino acids 1-603) was performed by using QuikChange Site-Directed Mutagenesis kit (Stratagene) or KOD-Plus Mutagenesis kit (Toyobo). Among amino acids 1-603, amino acids 214-235 were deleted or mutated to alamines (Figure 3D).

Immunoperoxidase staining and immunofluorescence imaging. Immunoperoxidase staining and immunofluorescence imaging were performed using patient or control sera (1:50-100 dilution) or a monoclonal antibody raised against amino acids 15-29 of CaSR (C0493, MilliporeSigma) (Figure 1B) or 214-235 of CaSR (MA1-934, Affinity BioReagents) (Figure 2B) or polyclonal anti-Flag antibody (F-7425, MilliporeSigma) (Figure 2B) (31, 72). Briefly, HEK293 cells expressing the human CaSR or Flag-tagged human CaSR were fixed in $100 \%$ methanol at $-20^{\circ} \mathrm{C}$ for 2 minutes to evaluate whole-cell expression (with permeabilization). To evaluate cell surface expression (without permeabilization), the cells were fixed at $4^{\circ} \mathrm{C}$ for 15 minutes in $4 \%$ paraformaldehyde/PBS. Bound IgG was detected by using either a peroxidase-conjugated, goat anti-human antiserum specific for the $\gamma$ chain of IgG (074-1002, Kirkegaard and Perry Laboratories) from the $\mathrm{AEC}^{+}$system or goat anti-human IgG tagged with Alexa Fluor 488 (green) (A-11013, Molecular Probes), goat anti-mouse IgG tagged with Alexa Fluor 488 (green) (A-11029, Molecular Probes), or Alexa Fluor 594 (red) (A-11032, Molecular Probes), anti-rabbit IgG tagged with Alexa Fluor 488 (green) (A-11034, Molecular Probes), or Alexa Fluor 568 (red) (A-11036, Molecular Probes) as described previously (31, 72). Fluorescence images were then collected by using an ECLIPSE E600 microscope (NIKON).

Measurement of CaSR-stimulated IP accumulation and ERK1/2 phosphorylation. Measurement of CaSR-stimulated IP accumulation and ERK1/2 phosphorylation was performed as described previously (31). For IP accumulation, HEK293 cells expressing the human CaSR were plated onto collagen-coated 24-well plates at $1.5 \times 10^{5}$ cells per well under DMEM with $0.3 \mathrm{mM} \mathrm{CaCl}_{2}$ and labeled with myo- $\left[{ }^{3} \mathrm{H}\right]$ inositol $(6 \mu \mathrm{Ci} / \mathrm{ml}$, Amersham Biosciences) for 24 hours. After washing with a calcium-free medium containing $5 \mathrm{mM} \mathrm{LiCl}$ for 10 minutes, the cells were incubated in the presence of $5 \mathrm{mM} \mathrm{LiCl}$ for 60 minutes. IP accumulation was estimated by determining the ratio of IP radioactivity to the sum of IP plus total inositol radioactivity. For 
determining ERK1/2 phosphorylation, the cells were plated onto 12-well plates and starved for 12-16 hours in DMEM with $0.3 \mathrm{mM} \mathrm{CaCl}_{2}$. The cells were then stimulated with various concentrations of calcium with or without purified IgG and the reactions were terminated. Protein extracts were then analyzed by Western blotting using monoclonal anti-phospho MAPK antibody (E10, \#9106, Cell Signaling).

Biotinylation of the cell surface forms of Flag-tagged CaSR and detection of CaSR dimerization. Prior to preparing whole-cell lysates, 293 Flag-tagged CaSR cells were rinsed twice with PBS and treated with $0.5 \mathrm{mg} / \mathrm{ml}$ EZ-link Sulfo-NHS-Biotin (Thermo Scientific) at room temperature with constant agitation for 30 minutes to biotinylate the proteins on the cell surface. The reaction was quenched by incubating the cells in $100 \mathrm{mM}$ glycine for 5 minutes. The surface-biotinylated cells were rinsed and solubilized with 1\% Triton-X, $0.5 \%$ NP-40, 150 mM NaCl, 10 mM Tris-HCl (pH 7.4), 2 mM EDTA, 1 mM EGTA, 100 mM iodoacetamide, and protease inhibitors, including aprotinin, leupeptin, PMSF, and pepstatin $(1 \times$ immunoprecipitation buffer) at $4^{\circ} \mathrm{C}$ for 45 minutes. After centrifugation (18,000 $\mathrm{g}$ for 3 minutes), the supernatants were placed in new tubes in which $1 \mathrm{mg}$ anti-Flag M2 monoclonal antibody (F3165, MilliporeSigma) was added with rotation at $4^{\circ} \mathrm{C}$ for 1 hour. After this, $15 \mu \mathrm{l}$ of protein $\mathrm{G}$ (Santa Cruz) was added to the mixture for an additional 45 minutes rotation at $4^{\circ} \mathrm{C}$. After washing, the immunoreactive species were eluted in $60 \mu 1$ of $2 \times$ electrophoresis sample buffer with or without $100 \mathrm{mM} \mathrm{DTT}$ at $50^{\circ} \mathrm{C}$ for 3 minutes. The sample was analyzed by Western blotting using horseradish peroxidase-labeled streptavidin (Kirkegaard and Perry Laboratories).

Cell surface ELISA. The cell surface expression of receptors in HEK293 cells expressing WT or mutant Flag-tagged human CaSR was quantified by ELISA. Briefly, 1 day after transfection, HEK293 cells were plated onto 24-well plates. After an additional 24 hours, the cells were washed and incubated with patient or control sera (1:50-1:100 dilution) for 1 hour at $4^{\circ} \mathrm{C}$. After washing, the cells were fixed at $4^{\circ} \mathrm{C}$ for 15 minutes in $4 \%$ formaldehyde/PBS (without permeabilization). The cells were then incubated in HRP-conjugated anti-human IgG solution (1:2000) at room temperature for 1 hour. After washing, the cells were treated with substrates (o-phenylenediamine dihydrochloride, MilliporeSigma) for 5 minutes at room temperature. This reaction was stopped by the addition of an equivalent volume of $2.5 \mathrm{~N} \mathrm{HCl}$, and the absorption levels were read at $492 \mathrm{~nm}$ using plate reader (EnSpire, Perkin Elmer).

Preparation of human parathyroid cells and determination of PTH secretion. Human parathyroid cells were prepared from parathyroid adenoma excised under approval of the Institutional Review Board of The University of Tokyo, as described previously (73) with some modifications. Briefly, parathyroid tissues were finely minced with scissors, digested for 60 minutes with Ham's F10 medium containing $1 \mathrm{mg} / \mathrm{ml}$ collagenase, and then passed through a nylon filter. Viable cells separated by Percoll gradient were used in the sequential assay. Parathyroid cells were plated into culture medium based on Ham's F10 containing 4\% heat-inactivated fetal bovine serum and $0.5 \mathrm{mM}$ calcium. After overnight culture to allow adherence to plates, cells were pretreated with $200 \mathrm{ng} / \mathrm{ml}$ PTX and washed twice to remove previously secreted PTH. Following 6-hour incubation with assay medium based on Ham's F10 with $0.2 \%$ bovine serum albumin containing identical concentration of calcium and patient's or control serum (1:20), media were collected and stored at $-80^{\circ} \mathrm{C}$ until immunoradiometric assay for whole PTH (DS Pharma Biomedical Cooperation).

Statistics. Two-sided two-tailed Student's $t$ test (for 2 comparisons) and two-sided Dunnett's/Tukey's multiple comparison procedures (for multiple comparisons) were used for statistical analysis. All analyses were performed using JMP 13 software. Averaged data from 3 independent experiments are shown, and error bars represent the SEM unless otherwise stated. $P<0.05$ was considered significant for all analyses.

Study approval. This study was performed under approval of the Institutional Review Board of The University of Tokyo (3680 and G1067), and written informed consent was received from our AHH patient prior to inclusion in this study.

\section{Author contributions}

N Makita, JS, K Manaka, and K Mitani performed the experiments; YK, TN, and TO prepared the experiment using parathyroid cells; N Makita, TA, MN, MO, YT, N Matsumoto, AK, and TI analyzed the data; $\mathrm{N}$ Makita and TI wrote the paper.

\section{Acknowledgments}

We thank members of Iiri/Makita lab for suggestions and assistance. We also thank Edward M. Brown for generously donating tagged CaSR construct. TI and N Makita are supported by a Grant-in-Aid for Scientific Research from the Ministry of Education, Science, Sports, Culture, Japan. 
Address correspondence to: Noriko Makita, 7-3-1 Hongo Bunkyo-ku, Tokyo, 113-8655 Japan; Phone: 81.3.3815.5411; E-mail: norimaki-tky@umin.ac.jp. Or to: Taroh Iiri, 2-16-1 Sugao, Miyamae-ku, Kawasaki, 216-8511 Japan; Phone: 81.3.3815.5411 or 81.44.977.8111; E-mail: tiiri-tky@umin.ac.jp or tiiri@marianna-u.ac.jp.

1. Kenakin T. New concepts in drug discovery: collateral efficacy and permissive antagonism. Nat Rev Drug Discov. 2005;4(11):919-927.

2. Hill SJ. G-protein-coupled receptors: past, present and future. Br J Pharmacol. 2006;147 Suppl 1:S27-S37.

3. Urban JD, et al. Functional selectivity and classical concepts of quantitative pharmacology. J Pharmacol Exp Ther. 2007;320(1):1-13.

4. Williams C, Hill SJ. GPCR signaling: understanding the pathway to successful drug discovery. Methods Mol Biol. 2009;552:39-50.

5. Audet M, Bouvier M. Restructuring G-protein- coupled receptor activation. Cell. 2012;151(1):14-23.

6. Kenakin T. What is pharmacological 'affinity'? Relevance to biased agonism and antagonism. Trends Pharmacol Sci. 2014;35(9):434-441

7. Violin JD, Crombie AL, Soergel DG, Lark MW. Biased ligands at G-protein-coupled receptors: promise and progress. Trends Pharmacol Sci. 2014;35(7):308-316

8. Wootten D, Christopoulos A, Marti-Solano M, Babu MM, Sexton PM. Mechanisms of signalling and biased agonism in G protein-coupled receptors. Nat Rev Mol Cell Biol. 2018;19(10):638-653.

9. Wootten D, Christopoulos A, Sexton PM. Emerging paradigms in GPCR allostery: implications for drug discovery. Nat Rev Drug Discov. 2013;12(8):630-644.

10. Christopoulos A. Advances in G protein-coupled receptor allostery: from function to structure. Mol Pharmacol. 2014;86(5):463-478

11. Khoury E, Clément S, Laporte SA. Allosteric and biased g protein-coupled receptor signaling regulation: potentials for new therapeutics. Front Endocrinol (Lausanne). 2014;5:68

12. Cook AE, et al. Biased allosteric modulation at the $\mathrm{CaS}$ receptor engendered by structurally diverse calcimimetics. Br J Pharmacol. 2015;172(1):185-200.

13. Smith JS, Lefkowitz RJ, Rajagopal S. Biased signalling: from simple switches to allosteric microprocessors. Nat Rev Drug Discov. 2018;17(4):243-260

14. Ross EM. Signal sorting and amplification through G protein-coupled receptors. Neuron. 1989;3(2):141-152.

15. Gether U. Uncovering molecular mechanisms involved in activation of G protein-coupled receptors. Endocr Rev. 2000;21(1):90-113.

16. Pierce KL, Premont RT, Lefkowitz RJ. Seven-transmembrane receptors. Nat Rev Mol Cell Biol. 2002;3(9):639-650.

17. van der Westhuizen ET, Valant C, Sexton PM, Christopoulos A. Endogenous allosteric modulators of G protein-coupled receptors. J Pharmacol Exp Ther. 2015;353(2):246-260.

18. Brown EM, et al. Cloning and characterization of an extracellular $\mathrm{Ca}(2+)$-sensing receptor from bovine parathyroid. Nature 1993;366(6455):575-580.

19. Brown EM, Vassilev PM, Quinn S, Hebert SC. G-protein-coupled, extracellular Ca(2+)-sensing receptor: a versatile regulator of diverse cellular functions. Vitam Horm. 1999;55:1-71.

20. Hofer AM, Brown EM. Extracellular calcium sensing and signalling. Nat Rev Mol Cell Biol. 2003;4(7):530-538.

21. Brown EM. Clinical lessons from the calcium-sensing receptor. Nat Clin Pract Endocrinol Metab. 2007;3(2):122-133.

22. Makita N, Iiri T. Calcium-sensing receptor. AfCS Nature Molecule Pages. https://www.doi.org/10.1038/mp.a004000.01. Published June 5, 2008. Accessed April 5, 2019.

23. Saidak Z, Brazier M, Kamel S, Mentaverri R. Agonists and allosteric modulators of the calcium-sensing receptor and their therapeutic applications. Mol Pharmacol. 2009;76(6):1131-1144.

24. Zhang C, et al. Molecular Basis of the Extracellular Ligands Mediated Signaling by the Calcium Sensing Receptor. Front Physiol. 2016;7:441.

25. Díaz-Soto G, Rocher A, García-Rodríguez C, Núñez L, Villalobos C. The Calcium-Sensing Receptor in Health and Disease. Int Rev Cell Mol Biol. 2016;327:321-369.

26. Pollak MR, et al. Mutations in the human $\mathrm{Ca}(2+)$-sensing receptor gene cause familial hypocalciuric hypercalcemia and neonatal severe hyperparathyroidism. Cell. 1993;75(7):1297-1303.

27. Brown EM. Anti-parathyroid and anti-calcium sensing receptor antibodies in autoimmune hypoparathyroidism. Endocrinol Metab Clin North Am. 2009;38(2):437-x.

28. Betterle C, Garelli S, Presotto F. Diagnosis and classification of autoimmune parathyroid disease. Autoimmun Rev. 2014;13(45):417-422.

29. Makita N, Iiri T. Biased agonism: a novel paradigm in G protein-coupled receptor signaling observed in acquired hypocalciuric hypercalcemia. Endocr J. 2014;61(4):303-309.

30. Pallais JC, Kifor O, Chen YB, Slovik D, Brown EM. Acquired hypocalciuric hypercalcemia due to autoantibodies against the calcium-sensing receptor. $N$ Engl J Med. 2004;351(4):362-369.

31. Makita N, et al. An acquired hypocalciuric hypercalcemia autoantibody induces allosteric transition among active human Ca-sensing receptor conformations. Proc Natl Acad Sci USA. 2007;104(13):5443-5448.

32. Pallais JC, et al. Autoimmune hypocalciuric hypercalcemia unresponsive to glucocorticoid therapy in a patient with blocking autoantibodies against the calcium-sensing receptor. J Clin Endocrinol Metab. 2011;96(3):672-680.

33. Conklin BR, Bourne HR. Homeostatic signals. Marriage of the flytrap and the serpent. Nature. 1994;367(6458):22.

34. Nesbit MA, et al. Mutations affecting G-protein subunit $\alpha 11$ in hypercalcemia and hypocalcemia. N Engl J Med. 2013;368(26):2476-2486.

35. Howles SA, et al. Cinacalcet corrects hypercalcemia in mice with an inactivating Ga11 mutation. JCI Insight. 2017;2(20):96540.

36. Davey AE, Leach K, Valant C, Conigrave AD, Sexton PM, Christopoulos A. Positive and negative allosteric modulators pro- 
mote biased signaling at the calcium-sensing receptor. Endocrinology. 2012;153(3):1232-1241.

37. Leach K, Gregory KJ. Molecular insights into allosteric modulation of Class C G protein-coupled receptors. Pharmacol Res. 2017;116:105-118.

38. Nemeth EF, et al. Calcimimetics with potent and selective activity on the parathyroid calcium receptor. Proc Natl Acad Sci USA 1998;95(7):4040-4045.

39. Kemp EH, et al. Mapping of human autoantibody binding sites on the calcium-sensing receptor. J Bone Miner Res. 2010;25(1):132-140

40. Pin JP, et al. Allosteric functioning of dimeric class C G-protein-coupled receptors. FEBS J. 2005;272(12):2947-2955.

41. Kniazeff J, Prézeau L, Rondard P, Pin JP, Goudet C. Dimers and beyond: The functional puzzles of class C GPCRs. Pharmacol Ther. 2011;130(1):9-25.

42. Urwyler S. Allosteric modulation of family C G-protein-coupled receptors: from molecular insights to therapeutic perspectives Pharmacol Rev. 2011;63(1):59-126.

43. Geng Y, et al. Structural mechanism of ligand activation in human calcium-sensing receptor. Elife. 2016;5:e13662.

44. Kuo E, Kemp EH, Sandhu HK, Brown EM, Weetman AP, Huang CL. Acquired hypocalciuric hypercalcemia in a patient with CKD. Am J Kidney Dis. 2013;62(6):1151-1154.

45. Marx SJ. Calcimimetic Use in Familial Hypocalciuric Hypercalcemia-A Perspective in Endocrinology. J Clin Endocrinol Metab. 2017;102(11):3933-3936.

46. Abusahmin H, Surya A, Aldridge A, Okosieme O, Das G. Cinacalcet: A Viable Therapeutic Option for Primary Hyperparathyroidism in the Elderly. Indian J Endocrinol Metab. 2018;22(4):485-488.

47. Dixon M, Luthra V, Todd C. Use of cinacalcet in lithium-induced hyperparathyroidism. BMJ Case Rep. 2018;2018:bcr-2018-225154.

48. Sun X, Huang L, Wu J, Tao Y, Yang F. Novel homozygous inactivating mutation of the calcium-sensing receptor gene in neonatal severe hyperparathyroidism responding to cinacalcet therapy: A case report and literature review. Medicine (Baltimore). 2018;97(45):e13128

49. Warady BA, et al. A randomized, double-blind, placebo-controlled study to assess the efficacy and safety of cinacalcet in pediatric patients with chronic kidney disease and secondary hyperparathyroidism receiving dialysis. Pediatr Nephrol. 2019;34(3):475-486.

50. Petrel C, Kessler A, Dauban P, Dodd RH, Rognan D, Ruat M. Positive and negative allosteric modulators of the Ca2+-sensing receptor interact within overlapping but not identical binding sites in the transmembrane domain. J Biol Chem. 2004;279(18):18990-18997.

51. Servant G, et al. Positive allosteric modulators of the human sweet taste receptor enhance sweet taste. Proc Natl Acad Sci USA. 2010;107(10):4746-4751.

52. Servant G, Tachdjian C, Li X, Karanewsky DS. The sweet taste of true synergy: positive allosteric modulation of the human sweet taste receptor. Trends Pharmacol Sci. 2011;32(11):631-636.

53. Kim SK, Chen Y, Abrol R, Goddard WA, Guthrie B. Activation mechanism of the G protein-coupled sweet receptor heterodimer with sweeteners and allosteric agonists. Proc Natl Acad Sci USA. 2017;114(10):2568-2573.

54. Pin JP, et al. The activation mechanism of class-C G-protein coupled receptors. Biol Cell. 2004;96(5):335-342.

55. Møller TC, Moreno-Delgado D, Pin JP, Kniazeff J. Class C G protein-coupled receptors: reviving old couples with new partners. Biophys Rep. 2017;3(4):57-63.

56. Wu H, et al. Structure of a class C GPCR metabotropic glutamate receptor 1 bound to an allosteric modulator. Science. 2014;344(6179):58-64.

57. Vafabakhsh R, Levitz J, Isacoff EY. Conformational dynamics of a class C G-protein-coupled receptor. Nature. 2015;524(7566):497-501.

58. Levitz J, Habrian C, Bharill S, Fu Z, Vafabakhsh R, Isacoff EY. Mechanism of Assembly and Cooperativity of Homomeric and Heteromeric Metabotropic Glutamate Receptors. Neuron. 2016;92(1):143-159.

59. Khan MA, Conigrave AD. Mechanisms of multimodal sensing by extracellular $\mathrm{Ca}(2+)$-sensing receptors: a domain-based survey of requirements for binding and signalling. Br J Pharmacol. 2010;159(5):1039-1050.

60. Mun HC, Culverston EL, Franks AH, Collyer CA, Clifton-Bligh RJ, Conigrave AD. A double mutation in the extracellular Ca2+-sensing receptor's Venus flytrap domain that selectively disables L-amino acid sensing. J Biol Chem. 2005;280(32):29067-29072.

61. Scholler P, et al. Allosteric nanobodies uncover a role of hippocampal mGlu2 receptor homodimers in contextual fear consolidation. Nat Commun. 2017;8(1):1967.

62. Thomsen AR, Smajilovic S, Bräuner-Osborne H. Novel strategies in drug discovery of the calcium-sensing receptor based on biased signaling. Curr Drug Targets. 2012;13(10):1324-1335.

63. Hannan FM, et al. A homozygous inactivating calcium-sensing receptor mutation, Pro339Thr, is associated with isolated primary hyperparathyroidism: correlation between location of mutations and severity of hypercalcaemia. Clin Endocrinol (Oxf). 2010;73(6):715-722.

64. Marx SJ. Hyperplasia in glands with hormone excess. Endocr Relat Cancer. 2016;23(1):R1-14.

65. Marx SJ. Familial Hypocalciuric Hypercalcemia as an Atypical Form of Primary Hyperparathyroidism. J Bone Miner Res. 2018;33(1):27-31

66. Kizilgul M, et al. The prognostic significance of glutamic acid decarboxylase antibodies in patients with chronic pancreatitis undergoing total pancreatectomy with islet autotransplantation. Diabetes Metab. 2018;null:null.

67. Makita N, et al. Human G(salpha) mutant causes pseudohypoparathyroidism type Ia/neonatal diarrhea, a potential cell-specific role of the palmitoylation cycle. Proc Natl Acad Sci USA. 2007;104(44):17424-17429.

68. Makita N, et al. Attenuated desensitization of $\beta$-adrenergic receptor by water-soluble $\mathrm{N}$-nitrosamines that induce S-nitrosylation without NO release. Circ Res. 2013;112(2):327-334

69. Iiri T, Herzmark P, Nakamoto JM, van Dop C, Bourne HR. Rapid GDP release from Gs alpha in patients with gain and loss of endocrine function. Nature. 1994;371(6493):164-168.

70. Iiri T, Bell SM, Baranski TJ, Fujita T, Bourne HR. A Gsalpha mutant designed to inhibit receptor signaling through Gs. Proc Natl Acad Sci USA. 1999;96(2):499-504.

71. Takahashi K, et al. V2 vasopressin receptor (V2R) mutations in partial nephrogenic diabetes insipidus highlight protean ago- 
nism of V2R antagonists. J Biol Chem. 2012;287(3):2099-2106.

72. Makita N, et al. Analysis of the V2 Vasopressin Receptor (V2R) Mutations Causing Partial Nephrogenic Diabetes Insipidus Highlights a Sustainable Signaling by a Non-peptide V2R Agonist. J Biol Chem. 2016;291(43):22460-22471.

73. Björklund P, Hellman P. Culture of parathyroid cells. Methods Mol Biol. 2012;806:43-53. 